\title{
AN UPDATED ECOLOGICAL ANALYSIS OF BRITISH MESOTHELIOMA RATES IN RELATION TO POTENTIAL
} EXPOSURE TO SIMIAN VIRUS 40 (SV40)

Andrew Darnton, John Hodgson Health \& Safety Executive, Bootle, UK

10.1136/oemed-2011-100382.195

Objectives Some laboratory studies have suggested that exposure to Simian Virus 40 (SV40) might be a risk factor for mesothelioma. Most human epidemiological studies have not shown an association, though have lacked statistical power because of limited follow-up. We tested the hypothesis of there being an association via an update of an earlier ecological analysis in Britain.

Methods National mesothelioma mortality for birth cohorts (1951-55 and 1956-60) with potential for exposure to SV40 via poliovirus vaccination in Britain were compared with those for a birth cohort (1962-66) likely to be unexposed, with consideration given to potential confounding by changes in asbestos exposure.

Results There was evidence that mesothelioma rates were higher in the first SV40 potentially exposed cohort than the unexposed in both men ( $\mathrm{RR}=2.4,95 \%$ CI 1.5 to 3.9 ) and women ( $R R=2.7,95 \%$ CI 1.1 to 6.1 . In the second SV40 potentially exposed cohort, rates in men were no higher than in the unexposed cohort ( $R R=1.0,95 \%$ CI 0.6 to 1.9) while rates in women were higher but not statistically significantly so $(R R=2.0,95 \%$ CI 0.8 to 4.8 ).

Conclusions The reduction in the RR for the second cohort comparison in males may reflect a reduction in asbestos exposure in the second SV40 potentially exposed cohort rather than an effect due to SV40. The suggestion of an increased $\mathrm{RR}$ based on both cohort comparisons in females could reflect an SV40 effect confined to females, or more likely, a later reduction in asbestos exposure among women due to a high proportion of their exposures arising from non-occupational sources. 